\title{
25 Research Square \\ Circulating Tumor Cells as a Biomarker for Precise Management of Lung Cancer
}

\section{Qian Li}

Nanjing Medical University

Chenyu Xu

Nanjing Medical University

Zhenhua Yang ( $\sim$ goat2007@163.com )

Nanjing Medical University

\section{Research Article}

Keywords: Lung cancer, biomarker, circulating tumor cells, precise management

Posted Date: June 16th, 2021

DOI: https://doi.org/10.21203/rs.3.rs-598253/v1

License: (1) This work is licensed under a Creative Commons Attribution 4.0 International License. Read Full License 


\section{Abstract}

Background: Circulating tumor cells (CTCs) with potential utility in cancer treatment provide a less invasive way to illustrate the pathogenesis of tumor. In this study, we evaluated the efficiency of CTCs in early diagnosis and/or precisely indication of lung cancer (LC) patients responding to chemotherapy.

Methods: Fifty-six patients newly diagnosed with LC were included in this study, while 42 patients with nonmalignant pulmonary disease were included as positive control group and 27 healthy individuals as control. CTCs were measured at baseline in all eligible subjects and after two cycles of chemotherapy in 25 advanced LC patients, with a strategy of negative enrichment combined with immunostainingfluorescence in situ hybridization (imFISH). The receiver operating characteristic (ROC) curve was plotted to determine the sensitivity and specificity of the CTC for the LC diagnosis. Kaplan-Meier curve and twosided log-rank test were used for univariate survival analysis.

Results: The number of CTCs was significantly increased in LC patients, compared with positive control and healthy controls. The sensitivity and specificity were $87.5 \%$ and $95.7 \%$, with a cutoff value of 2 cells/3.2ml (AUC=0.975, 95\% Cl, 0.953-0.998, $p=0.001)$. Moreover, the sensitivity of CTCs was much higher than individual or the combination of serum tumor markers (NSE/CYFRA21-1/CEA), especially in the early diagnosis of LC. Twenty-five advanced LC patients were subsequently followed up for an average of 18.2 months (6-36 months). Decline of CTC count after two cycles of chemotherapy was highly predictive for progression free survival (PFS) (13.5 vs. 8.5 months; $p=0.025$ ) and overall survival (OS) (27.6 vs. 16.8 months; $p=0.009$ ). Among the patients classified as stable disease (SD) based on post-treatment CT scan after two cycles of chemotherapy, there was significant elevation in the PFS (14.0 vs. 8.7 months; $p=0.033$ ) and OS ( 25.8 vs. 15.9 months; $p=0.018)$ in those with decreased CTC number compared with those presenting stable or elevated CTCs number.

Conclusions: CTCs may act as precise biomarker for early detection and therapy monitoring of LC. It may serve as good compensation for imaging.

\section{Background}

Lung cancer (LC), a malignant tumor of uncontrolled cell growth metastasizing in distant tissues, is the most leading cause of cancer related death according to the 2012 GLOBOCAN [1]. There is a large variation in the clinical outcome of LC patients depending on staging upon diagnosis. To be specific, the 5 -year relative survival rate was approximately $55 \%$ for those with localized lesion, $28 \%$ for regional disease, and merely $4 \%$ for distant metastatic LC, respectively. To our best knowledge, the majority of LC patients (57\%) show metastasis at initial diagnosis [2]. Standard chemotherapy contributes to the improvement of prognosis in these patients; however, the prediction of treatment outcome is somehow affected by sensitivity of current imaging techniques, especially in the presence of slight changes in solid tumor. Consequently, it is urgent to develop sensitive and predictive biomarkers for early diagnosis and dynamic management of LC patients. Circulating tumor cells (CTCs), defined as tumor cells shed 
from solid tumors entering the peripheral bloodstream, provide a less invasive way to inquire the tumor information [3]. There are strong evidences that CTCs are disseminated in the circulation even before the appearance of visible tumors by imaging technique [4-6]. On this basis, detection of CTCs in the peripheral blood is feasible for early detection and monitoring of solid cancer. In fact, CTCs have been recommended as a novel prognostic marker for breast cancer according to the Eighth Edition of the AJCC Cancer Staging Manual [7]. However, its sensitivity for CTC detection is limited as CTC may lose epithelial cell adhesion molecule (EPCAM) and Cytokeratin (CK) during epithelial mesenchymal transition [8, 9]. Under this circumstance, aneuploidy detection serving as a common feature of cancer may provide a fresh perspective for CTC detection $[10,11]$.

In this study, CTCs were determined in blood samples collected from LC patients, patients with nonmalignant pulmonary disease and normal control. We aimed to investigate whether CTCs could provide early diagnostic information and/or precise indication of response in LC patients after chemotherapy.

\section{Material And Methods}

\section{Subjects}

Fifty-six patients newly diagnosed with LC (male: 38; female: 18; median age: 65.3yrs) admitted into our hospital from July 2014 to December 2016 were included in this study. Written informed consent was obtained from each patient. The study protocols were approved by the Ethics Committee of Nanjing First Hospital, Nanjing Medical University. Patients with no history of malignancy within 5 years received no treatment were eligible for this study. In addition, 27 matched healthy donors (male: 12; female: 15; median age: 55.8 yrs) served as control. Meanwhile, 42 patients (male:25; female: 17; median age: $55.4 y r s$ ) with nonmalignant pulmonary diseases (e.g. pneumonia, pulmonary tuberculosis, pulmonary hamartoma and pneumonectasis) were recruited as positive control. Written informed consent was obtained from each subject.

\section{CTC analysis by negative enrichment combined with immunostaining-fluorescence in situ hybridization (imFISH)}

Strategies for CTC enrichment and identification in each group were established according to the previous description [12]. Briefly, peripheral blood $(3.2 \mathrm{ml})$ was collected from each subject into acid citrate dextrose (ACD) - anticoagulant tubes (Becton, Dickinson and Company, Franklin Lake, NJ, USA), followed by plasma isolation based on centrifugation. The red blood cells were then lysed with CS2 buffer (Cyttel, Jiangsu, China). Subsequently, the residual cell particles were resuspended in CS1 buffer (Cyttel), and then were incubated with anti-CD45 antibody conjugated magnetic beads (Cyttel).

Afterwards, the magnetic beads were separated using a magnetic stand (Promega, Madison, WI, USA), and the solution containing CTCs were smeared onto slides for identification. 
The generated cells after the above procedures were fixed with CF2 (Cyttel) for $8 \mathrm{~min}$. Then the sliders were immerged in saline-sodium citrate (SSC) buffer at $37^{\circ} \mathrm{C}$ for $10 \mathrm{~min}$ and dehydrated by gradient ethanol (75\%, $85 \%$ and $100 \%, 2$ min for each concentration). Hybridization solution containing centromere probe 8 (CEP8) was added to the slides and incubated in hybridizer (DAKO) for $90 \mathrm{~min}$. Subsequently, the cells were immunostained with anti-CD 45 antibody conjugated to Alexa Fluor 594 at room temperature for $1 \mathrm{hr}$. After adding nuclear dye 4, 6-diamidino-2-phenylindole (DAPI, Invitrogen), slides were mounted for image analysis under a fluorescence microscope. CTCs positivity was defined as presence of hyperdiploid and a phenotype of CEP8+/DAPI+/CD45-.

\section{Efficiency of CTC in distinguishing LC}

To investigate the efficiency of CTCs expression in distinguishing LC, we compared the CTCs expression in LC patients with that of the normal control $(n=27)$, as well as the patients with nonmalignant pulmonary diseases $(n=42)$. The expression CTCs was determined using the $\mathrm{mFISH}$ technique according to the previous description [13].

\section{Comparison of CTCs with serum tumor markers in detection $L C$}

For the serum tumor marker detection, peripheral blood $(3 \mathrm{~mL})$ was collected into tubes without anticoagulants at the initial diagnosis of LC from 56 LC patients. The samples were centrifuged at $1,500 \mathrm{~g}$ for $10 \mathrm{~min}$, and the upper layer of the serum was subject to an automatic immunoassay analyzer (Cobas e601, Roche, Indianapolis, IN), to determine the expression of CEA, CYFRA21-1 and NSE. According to the manufacturer's instructions, the normal reference value of the three serum tumor markers was $0-6 \mathrm{ng} / \mathrm{ml}$ for CEA, 0-6.9 ng/ml for CYFRA21-1 and 0-16.3 ng/ml for NSE, respectively. Positivity was considered upon exceeding the upper limit of the normal threshold. A comparison was held for the screening rate of LC based on the efficiency of CTC and these three markers.

\section{Correlation between CTCs and progression free survival (PFS) and overall survival (OS)}

Peripheral blood was collected at initial diagnosis from each patient. Twenty-five patients with advanced LC who only received systemic chemotherapy participated in the follow-up. Samples from these twentyfive patients were also collected one day before the third cycle of first-line chemotherapy. In addition, the clinical data including gender, age, histology, tumor stage and Ki-67 expression in primary tumor were collected. The clinical response of patients to chemotherapy was evaluated by CT scan according to the Response Evaluation Criteria in Solid Tumors (RECIST). PFS and OS were defined as the elapsed time from initial diagnosis to progression or even death. 


\section{Statistical analysis}

Data analysis was performed by SPSS 22.0 software (SPSS, Chicago, IL, USA). The receiver operating characteristic (ROC) curve was plotted to determine the diagnostic value of CTCs. Chi-square test or Fisher's exact test was utilized to investigate the correlation between CTC detection rate and the clinicopathological characteristics of patients. Kaplan-Meier curve and Log-rank test were used for survival analyses. $P$ value of less than 0.05 was statistically significant. All statistical tests were twosided. Graphs were plotted using the GraphPad Prism 6 software (GraphPad Software, Inc, USA).

\section{Results}

\section{Patient characteristics}

In total, 56 patients (male: 38 ; female: 18) with an average age of $65.3 \mathrm{yrs}$ (37-78 yrs) were included in this study. For the histological type, 45 (80.35\%) were diagnosed with non-small cell lung cancer (NSCLC), including 13 squamous cell carcinoma (SCC), 29 adenocarcinoma and 3 large cell lung cancer. The other 11 (19.65\%) were diagnosed with small cell lung cancer (SCLC). According to the cancer staging system proposed by American Joint Committee on Cancer (AJCC, 7 th Edition), 5 cases were at stage I, 10 at stage II, 11 at stage III, and 30 at stage IV, respectively. For the T, N and M staging, 5 cases were at stage T1, 18 at stage T2,12 at stage T3, and 21 at stage T4, respectively. Meanwhile, 8 patients were classified into stage N0 and 48 patients were classified into stage N1-N3, respectively. Thirty cases showed distant metastasis, while the other twenty-six were free of distant metastasis. Twenty-eight cases (50\%) patients showed vascular invasion. Immunohistochemical analysis indicated high expression of Ki67 ( $\geq 50 \%)$ in the primary tumor tissues in 16 cases, while the other 40 showed low expression $(<50 \%)$.

\section{Detection of CTCs in LC and controls}

Cells isolated from the peripheral blood were identified by the signals of DAPI, CEP8 and CD 45. CTCs were characterized as nucleated cells expressing CEP8 other than CD45. On this basis, CTCs (1-22 cells $/ 3.2 \mathrm{ml}$ ) were identified in all the LC patients $(100 \%)$ at baseline. In contrast, CTCs were detected in peripheral blood of three healthy individuals (11.11\%) and the count was 1 cell/3.2ml. The detection rate of CTCs in patients with nonmalignant pulmonary disease was $23.81 \%$. The CTC detection rate and number in LC group was significantly higher than those in nonmalignant and healthy controls $(p<0.05)$. According to the Youden's index, a cutoff value of 2 cells/3.2ml yielded a sensitivity of $87.5 \%(49 / 56)$ and a specificity of $95.7 \%$ (AUC=0.975, 95\% Cl, 0.953-0.998, $p<0.001$, Fig.1).

\section{Detection sensitivity of CTCs and serum biomarkers in LC patients}


To date, CEA, CYFRA21-1 and NSE had been suggested as candidate biomarkers for diagnosis and treatment of NSCLC or SCLC. In this study, we compared the sensitivity of CTCs and three types of serum tumor markers. Among the three serum tumor markers, CYFRA21-1 showed the highest sensitivity $(69.64 \%)$ among the 56 LC patients, followed by NSE (41.07\%) and CEA (39.29\%, Table 1$)$. The sensitivity was improved when all three tumor markers were utilized in a combined manner, resulting a sensitivity of up to $78.57 \%$. The sensitivity of CTCs (87.5\%) was much higher than the sensitivity of these markers used in a single or combined manner (Table 1).

To investigate the roles of CTCs and serum tumor markers in early diagnosis of LC, we analyzed their sensitivities according to pathological stage. As shown in Fig. 2, CYFRA21-1 ( $\geq 3.3 \mathrm{ng} / \mathrm{ml})$ was detected in 2 cases (40.0\%) at stage I, $3(30.0 \%)$ at stage II, $8(72.7 \%)$ of stage III, and $26(86.7 \%)$ at stage IV, respectively. Its sensitivity was significantly higher than that of CEA and NSE at any pathological stage. In the combination of these three serum biomarkers, the sensitivity showed slight improvement, with $3(60.0 \%)$ in stage I, $5(50.0 \%)$ in stage II, $9(81.8 \%)$ in stage III and 27 (90.0\%) in stage IV. Notably, the positive rate of CTCs was $80 \%$ in stage I and $90.0 \%$ in stage II, which was much higher than CEA, CYFRA21-1 and NSE. This indicated that CTCs were potentially beneficial in early diagnosis of LC.

\section{Correlation between CTCs and clinicopathological characteristics}

Table 2 showed the relationship between CTC positive rate and various clinicopathological characteristics. CTCs positivity was detected in $90 \%$ of patients with lymph node metastasis. It was slightly higher than that in patients without lymph node metastasis (75\%), despite no statistics difference $(p=0.259)$. In addition, no obvious correlation was found between CTC sensitivity and other clinicopathological characteristics, including age, gender, histology, vascular invasion, TNM stage, serum tumor markers and Ki-67 expression.

\section{Roles of CTCs in monitoring the efficiency of chemotherapy for advanced LC}

To investigate the monitoring efficiency of imaging test and CTCs examination, CT scanning and CTCs tests were performed in 25 advance LC patients before and after two cycles of chemotherapy. The evaluation of chemotherapy efficiency was often carried out after two cycles of chemotherapy. Twentyone showed stable disease (SD) and the other four showed partial response (PR), respectively. No statistical difference was noticed in the median CTCs count after chemotherapy compared with the baseline level ( 3 cells $/ 3.2 \mathrm{ml}$ vs. 5 cells $/ 3.2 \mathrm{ml}, p=0.204$ ). The CTCs count showed decline in 2 patients with PR and 10 patients with SD after chemotherapy, while it showed increase in 8 patients with SD and 1 patient with PR, and unchanged in 4 other patients including three of SD and one of PR. These indicated that CTC changes were not always consistent with radiological response. 
In this study, we also investigated the prognostic value of CTC on PFS and OS in LC patients, especially those with SD after chemotherapy. The patients were followed up until May 7, 2018, with an average follow-up period of 18.2 months (range 6-36 months). All patients experienced disease progression, among which 16 patients $(64 \%)$ died. There was no significant correlation between the CTCs count at baseline level and PFS or OS (data not shown). There was no significant correlation between the CTCs count at baseline level and PFS or OS (data not shown). According to the CTCs changes, patients were divided into favorable group with decrease of CTCs after chemotherapy and unfavorable group with increased or unchanged CTCs after chemotherapy. The median PFS in the favorable group was significantly higher than that of unfavorable group (13.5 months vs. 8.5 months, log-rank test, $p=0.025)$. Moreover, patients in favorable group also showed a significant longer median OS (27.6 months, $95 \% \mathrm{Cl}$ : 20.8-34.3 months) compared with those in the unfavorable group (16.8 months, $95 \%$ Cl: 13.4-20.3 months, log-rank $p=0.009)$. Interestingly, in the patients with SD as revealed by post-treatment CT, changes in CTC count were highly predictive for PFS ( 14.0 vs. 8.7 months; log rank test $p=0.033$, Fig. $3 \mathrm{~A}$ ) and $0 S$ ( 25.8 vs. 15.9 months; log rank, $p=0.018$, Fig.3B).

\section{Discussion}

CTCs detection is a non-invasive approach with potential utility in clinical practice, including early diagnosis, relapse prediction, prognosis and therapy monitoring for solid tumors. Therefore, it is necessary to establishing a sensitive and reliable CTCs detection method.

To date, several strategies have been developed for CTCs detection, including CellSearch, isolation method by size of epithelial tumor cells (ISET) and CTC-chip, have been reported in LC. The CellSearch system, the only method approved by the FDA, is classified as positive enrichment relying on an immune nanomagnetism bead-based capture to enrich the CTC population expressing EpCAM. In a previous study, Krebs et al. reported that CTCs detected by CellSearch was the strongest predictor of OS, but only $32 \%$ of metastatic NSCLC patients showed a level of $\geq 2 \mathrm{CTCs} / 7.5 \mathrm{~m}$ in blood. Additionally, CTCs were even undetectable in patients with stage IIIA NSCLC.[14] Hofman et al. reported increased sensitivity of CTCs detection in NSCLC in when combing the ISET with the CellSearch system in parallel (69\%) compared with the single application of ISET (50\%) and CellSearch (39\%) [15]. With the introduction of microfuidic platform into the CTCs detection, the sensitivity showed continuous improvement in the recent years. Moreover, Murlidhar et al [16]. reported that CTCs were detected in $77.8 \%$ of early LC patients at a clinical stage of I-III using the OncoBean Chip.

Nowadays, the combination of negative enrichment and imFISH, a strategy involving both epithelial and mesenchymal tumor cells detectable, showed great potential for CTC detection as it yielded a sensitivity of $76.2 \%, 69.4 \%$ and $75.8 \%$ for ovarian, ampullary and pancreatic cancer, respectively [17-19]. Based on the combination of negative enrichment and imFISH, two studies from Peking Union Medical College revealed that CTCs were detected in $84.25 \%$ or $87 \%$ of advanced NSCLC, which provided valuable prognostic information for NSCLC $[12,20]$. In this study, LC patients at early stages were also included. The sensitivity for CTCs detection was $87.5 \%$ in all patients and $80 \%$ in early LC patients, which was more 
sensitive than other methods used in our study and the three serum tumor markers. Taken together, CTCs identified by such method showed a moderate ability in diagnosis of LC, especially for the early detection.

Cancer cells have the potency of metastasis to other organs through circulation. It has been well acknowledged that metastasis is the major cause for cancer-related death. Cancer cells would invade into the peripheral tissues of the primary cancer cells, and then entered the blood circulation and lymphatic system, leading to formation of CTCs and distal metastasis. Subsequently, there would be exudation that may be adaptive to the microenvironment, forming metastatic lesions after dissemination, proliferation and colonization. Therefore, early screening of blood CTCs contributed to the evaluation of prognosis, efficiency and individual treatment. In a prospective study, patients with a high risk of COPD induced by cigarette-smoking may develop lung cancer only CTCs were detected in the follow-up [21]. In this study, three cases showed CTCs positivity in control group, while imaging technique showed no occupying lesions. Besides, there was no elevation in the cancer-related biomarkers. This indicated that there might be systemic malignancies in those patients, which needed further validation in the follow-up.

In a previous study, CTCs had been confirmed as an independent factor for PFS and OS [1]. After chemotherapy, there would be a time interval of 3 months to evaluate the treatment efficiency. There would be significant changes in the tumor size about 3 months later. Under most conditions, the tumor size would increase about 3 months later. This was really a delay of 3 months for the treatment. Indeed, this was really important for the treatment. with the development of CTCs, we could determine the CTC number. In cases of significant decline of CTCs, this indicated the efficiency of chemotherapy. The increase of CTC demonstrated that a poor efficiency, while new treatment regimens should be selected accordingly $[14,22]$.

For advanced LC patients not suitable for surgery, systemic chemotherapy is one of the standard treatment options. To date, the tumor response to chemotherapy was usually assessed by RECIST criterion, which was based on the change of tumor volume. However, increasing evidence suggests that this criterion may not always indicate therapeutic effect and do not adequately capture cancer biology [23]. Thus, alternate response metrics are required to evaluate the treatment efficiency and the survival rates. To our best knowledge, extensive studies have been carried out to explore the potential of CTCs in characterizing treatment efficacy. Meanwhile, two studies indicated that $[14,24]$ CTCs detected by CellSearch at baseline were the strongest prognostic factor for survival. According to RECIST, changes in CTC number after chemotherapy were not always consistent with treatment response, however, they were highly predictive for PFS and OS in NSCLC or SCLC. Similar conclusion was drawn in a previous study involved four kinds of lung cancer cell lines [25]. Recently, a prospective multicentric study reported that early CTC changes after therapy were not correlated with RECIST response in advanced cancer including LC [26]. Nevertheless, Hirose et al.[27] found that CTCs were significantly relevant to the rate of progressive disease in cytotoxic chemotherapy rather than survival.

In this study, changes of CTCs were highly related to PFS and OS, which were identical with the results obtained based on the same CTC detection strategy [20] and previous results by CellSearch [13]. It 
was noteworthy that the same trend was also noticed in the patients with SD. To our knowledge, this is the only study using a predictive model for accurately prognostic stratification in advance LC patients with the same RECIST response. Theoretically, the systemic therapy of cancer is relied on two aspects, including inhibiting the growth of the solid tumor and eradication of CTCs. It has been well acknowledged that radiological response plays important roles in evaluating the treatment efficiency of solid tumor and the survival rate. Surprisingly, the second node is totally ignored by current chemotherapy assessments. The precise enumeration of CTCs in peripheral blood may provide a more accurate predictor when combining with imaging technique. Furthermore, further molecular and functional analysis of CTCs also has great clinical potential in predicting and monitoring chemotherapy in LC patients [28-31].

There are some limitations in this study. The current study was performed at a single center with limited sample size, which may limit the power of statistical analysis. Additionally, the molecular mechanisms of CTCs were not further characterized to understand the biology of tumor cells in blood stream. In future, additional large scale, comprehensive studies are required to elucidate the roles of CTCs in the clinical management of LC.

\section{Conclusion}

In summary, CTCs was highly expressed in the patients with pulmonary cancer. In addition, there was a positive correlation between CTCs count and the severity of cancer. CTCs were crucial for the evaluation of OS and PFS in patients with advanced malignancy. Meanwhile, the count of peripheral CTCs combined with the imaging technique contributed to the diagnosis of the lung cancer.

\section{Abbreviations}

Circulating tumor cells (CTCs); lung cancer (LC); immunostaining-fluorescence in situ hybridization (imFISH); receiver operating characteristic (ROC); progression free survival (PFS); stable disease (SD); epithelial cell adhesion molecule (EPCAM); Cytokeratin (CK); acid citrate dextrose (ACD); saline-sodium citrate (SSC); containing centromere probe 8 (CEP8); progression free survival (PFS); overall survival (OS); Response Evaluation Criteria in Solid Tumors (RECIST); non-small cell lung cancer (NSCLC); squamous cell carcinoma (SCC); small cell lung cancer (SCLC); stable disease (SD); partial response (PR).

\section{Declarations}

\section{Ethics approval and consent to participate}

The study was approved by the Ethics Committee of Nanjing First Hospital and was conducted in accordance with the principles expressed in the Helsinki Declaration. Written informed consent was obtained from the participants.

\section{Consent for publication}


Not applicable.

\section{Availability of data and materials}

The datasets used and analysed during the current study are available from the corresponding author on reasonable request.

\section{Competing interests}

The authors declare that they have no competing interests.

\section{Funding}

None.

\section{Authors' contributions}

LQ drafted the article and made contributions to the conception and design of the study; XCY did the acquisition of data, or analysis and interpretation of data; $\mathrm{YZH}$ revised the manuscript. All authors reviewed the manuscript.

\section{Acknowledgements}

Not applicable.

\section{References}

1. Bidard FC, Peeters DJ, Fehm T, Nolé F, Gisbert-Criado R, Mavroudis D, et al. Clinical validity of circulating tumour cells in patients with metastatic breast cancer: a pooled analysis of individual patient data. Lancet Oncol. 2014;15:406-14.

2. Siegel RL MK, Jemal A. Cancer Statistics, 2017. A Cancer Journal for Clinicians. 2017;67:7-30.

3. Masuda T, Hayashi N, Iguchi T, Ito S, Eguchi H, Mimori K. Clinical and biological significance of circulating tumor cells in cancer. Mol Oncol. 2016;10:408-17.

4. Kim YR, Yoo JK, Jeong CW, Choi JW. Selective killing of circulating tumor cells prevents metastasis and extends survival. J Hematol Oncol. 2018;11:114.

5. Hosseini H, Obradović MMS, Hoffmann M, Harper KL, Sosa MS, Werner-Klein M, et al. Early dissemination seeds metastasis in breast cancer. Nature. 2016;540:552-8.

6. Massagué J, Obenauf AC. Metastatic colonization by circulating tumour cells. Nature. 2016;529:298306. 
7. Giuliano AE, Edge SB, Hortobagyi GN. Eighth Edition of the AJCC Cancer Staging Manual: Breast Cancer. Ann Surg Oncol. 2018;25:1783-5.

8. Buscail E, Chiche L, Laurent C, Vendrely V, Denost Q, Denis J, et al. Tumor-proximal liquid biopsy to improve diagnostic and prognostic performances of circulating tumor cells. Mol Oncol. 2019;13:1811-26.

9. Okuda K, Nakanishi R. Circulating Cancer-Associated Cells for the Early Detection and Decision-Making Concerning Effective Therapeutic Strategies for NSCLC. J Thorac Oncol. 2020;15:1397-8.

10. Lei Y, Sun N, Zhang G, Liu C, Lu Z, Huang J, et al. Combined detection of aneuploid circulating tumorderived endothelial cells and circulating tumor cells may improve diagnosis of early stage non-small-cell lung cancer. Clin Transl Med. 2020;10:e128.

11. Yang Y, Li J, Jin L, Wang D, Zhang J, Wang J, et al. Independent Correlation Between Ki67 Index and Circulating Tumor Cells in the Diagnosis of Colorectal Cancer. Anticancer Res. 2017;37:4693-700.

12. Zhang Z, Xiao Y, Zhao J, Chen M, Xu Y, Zhong W, et al. Relationship between circulating tumour cell count and prognosis following chemotherapy in patients with advanced non-small-cell lung cancer. Respirology. 2016;21:519-25.

13. Castro-Giner F, Aceto N. Tracking cancer progression: from circulating tumor cells to metastasis. Genome Med. 2020;12:31.

14. Krebs MG, Sloane R, Priest L, Lancashire L, Hou JM, Greystoke A, et al. Evaluation and prognostic significance of circulating tumor cells in patients with non-small-cell lung cancer. J Clin Oncol. 2011;29:1556-63.

15. Hofman V, llie MI, Long E, Selva E, Bonnetaud C, Molina T, et al. Detection of circulating tumor cells as a prognostic factor in patients undergoing radical surgery for non-small-cell lung carcinoma: comparison of the efficacy of the CellSearch Assay ${ }^{\mathrm{TM}}$ and the isolation by size of epithelial tumor cell method. Int $J$ Cancer. 2011;129:1651-60.

16. Murlidhar V, Reddy RM, Fouladdel S, Zhao L, Ishikawa MK, Grabauskiene S, et al. Poor Prognosis Indicated by Venous Circulating Tumor Cell Clusters in Early-Stage Lung Cancers. Cancer Res. 2017;77:5194-206.

17. Li Y, Ma G, Zhao P, Fu R, Gao L, Jiang X, et al. Improvement of sensitive and specific detection of circulating tumor cells using negative enrichment and immunostaining-FISH. Clin Chim Acta. 2018;485:95-102.

18. Liu H, Sun B, Wang S, Liu C, Lu Y, Li D, et al. Circulating Tumor Cells as a Biomarker in Pancreatic Ductal Adenocarcinoma. Cell Physiol Biochem. 2017;42:373-82. 
19. Sun B, Liu H, Wang S, Xiang J, Liu X. Prognostic impact of circulating tumor cells in patients with ampullary cancer. J Cell Physiol. 2018;233:5014-22.

20. Tong $B, X u Y$, Zhao J, Chen M, Xing J, Zhong W, et al. Prognostic significance of circulating tumor cells in non-small cell lung cancer patients undergoing chemotherapy. Oncotarget. 2017;8:86615-24.

21. Ilie M, Hofman V, Long-Mira E, Selva E, Vignaud JM, Padovani B, et al. "Sentinel" circulating tumor cells allow early diagnosis of lung cancer in patients with chronic obstructive pulmonary disease. PLoS One. 2014;9:e111597.

22. Hou JM, Krebs MG, Lancashire L, Sloane R, Backen A, Swain RK, et al. Clinical significance and molecular characteristics of circulating tumor cells and circulating tumor microemboli in patients with small-cell lung cancer. J Clin Oncol. 2012;30:525-32.

23. Johnson K, Gomez A, Burton J, White D, Chakravarty A, Schmid A, et al. Directional inconsistency between Response Evaluation Criteria in Solid Tumors (RECIST) time to progression and response speed and depth. Eur J Cancer. 2019;109:196-203.

24. Tamminga M, de Wit S, Schuuring E, Timens W, Terstappen L, Hiltermann TJN, et al. Circulating tumor cells in lung cancer are prognostic and predictive for worse tumor response in both targeted- and chemotherapy. Transl Lung Cancer Res. 2019;8:854-61.

25. Muinelo-Romay L, Vieito M, Abalo A, Nocelo MA, Barón F, Anido U, et al. Evaluation of Circulating Tumor Cells and Related Events as Prognostic Factors and Surrogate Biomarkers in Advanced NSCLC Patients Receiving First-Line Systemic Treatment. Cancers (Basel). 2014;6:153-65.

26. Massard C, Borget I, Farace F, Aspeslagh S, Le Deley MC, Le Tourneau C, et al. RECIST response and variation of circulating tumour cells in phase 1 trials: A prospective multicentric study. Eur $\mathrm{J}$ Cancer. 2017;83:185-93.

27. Hirose T, Murata Y, Oki Y, Sugiyama T, Kusumoto S, Ishida H, et al. Relationship of circulating tumor cells to the effectiveness of cytotoxic chemotherapy in patients with metastatic non-small-cell lung cancer. Oncol Res. 2012;20:131-7.

28. Nicolson MC, Fennell DA, Ferry D, O'Byrne K, Shah R, Potter V, et al. Thymidylate synthase expression and outcome of patients receiving pemetrexed for advanced nonsquamous non-small-cell lung cancer in a prospective blinded assessment phase II clinical trial. J Thorac Oncol. 2013;8:930-9.

29. Carter L, Rothwell DG, Mesquita B, Smowton C, Leong HS, Fernandez-Gutierrez F, et al. Molecular analysis of circulating tumor cells identifies distinct copy-number profiles in patients with chemosensitive and chemorefractory small-cell lung cancer. Nat Med. 2017;23:114-9.

30. Habli Z, AlChamaa W, Saab R, Kadara H, Khraiche ML. Circulating Tumor Cell Detection Technologies and Clinical Utility: Challenges and Opportunities. Cancers (Basel). 2020;12. 
31. Drapkin BJ, George J, Christensen CL, Mino-Kenudson M, Dries R, Sundaresan T, et al. Genomic and Functional Fidelity of Small Cell Lung Cancer Patient-Derived Xenografts. Cancer Discov. 2018;8:600-15.

\section{Tables}

Table 1. Detection sensitivity of CTCs and serum biomarkers in LC patients

\begin{tabular}{|lll|}
\hline Markers & \multicolumn{2}{c|}{ Positive } \\
\cline { 2 - 3 } & N & Rate \\
\hline CTC $\geq 1$ & 56 & $100.00 \%$ \\
\hline CTC $\geq 2$ & 49 & $87.50 \%$ \\
\hline NSE $\geq 16.3 n g / m l$ & 23 & $41.07 \%$ \\
\hline CYFRA21-1 $\geq 3.3 n g / m l$ & 39 & $69.64 \%$ \\
\hline CEA $\geq 6 n g / m l$ & 22 & $39.29 \%$ \\
\hline NSE+CYFRA21-1+CEA & 44 & $78.57 \%$ \\
\hline
\end{tabular}

Table 2. Association of CTCs with clinical pathological characteristics 


\begin{tabular}{|c|c|c|c|c|c|}
\hline \multirow[t]{2}{*}{ Variables } & & \multicolumn{3}{|c|}{ CTC } & \multirow[b]{2}{*}{$p$ value } \\
\hline & & $\mathbf{N}$ & Negative & Positive & \\
\hline \multirow[t]{2}{*}{ Age, years } & $<65$ & 27 & 4 & 23 & \multirow[t]{2}{*}{0.700} \\
\hline & $\geq 65$ & 29 & 3 & 26 & \\
\hline \multirow[t]{2}{*}{ Gender } & Female & 18 & 3 & 15 & \multirow[t]{2}{*}{0.669} \\
\hline & Male & 38 & 4 & 34 & \\
\hline \multirow[t]{2}{*}{ Histology } & NSCLC & 45 & 6 & 39 & \multirow[t]{2}{*}{1.000} \\
\hline & SCLC & 11 & 1 & 10 & \\
\hline \multirow[t]{4}{*}{ Depth of invasion } & $\mathrm{T} 1$ & 5 & 1 & 4 & \multirow[t]{4}{*}{0.109} \\
\hline & $\mathrm{T} 2$ & 18 & 0 & 18 & \\
\hline & T3 & 12 & 3 & 9 & \\
\hline & $\mathrm{T} 4$ & 21 & 3 & 18 & \\
\hline \multirow[t]{2}{*}{ Lymph node metastasis } & No & 8 & 2 & 6 & \multirow[t]{2}{*}{0.259} \\
\hline & N1 N3 & 48 & 5 & 43 & \\
\hline \multirow[t]{2}{*}{ Distant metastasis } & M0 & 26 & 3 & 23 & \multirow[t]{2}{*}{1.000} \\
\hline & M1 & 30 & 4 & 26 & \\
\hline \multirow[t]{4}{*}{ TNM stage } & 1 & 5 & 1 & 4 & \multirow[t]{4}{*}{0.932} \\
\hline & $\|$ & 10 & 1 & 9 & \\
\hline & III & 11 & 1 & 10 & \\
\hline & IV & 30 & 4 & 26 & \\
\hline \multirow[t]{2}{*}{ Vascular invasion } & No & 28 & 3 & 25 & \multirow[t]{2}{*}{1.000} \\
\hline & Yes & 28 & 4 & 24 & \\
\hline \multirow[t]{2}{*}{$\mathrm{CEA}, \mathrm{ng} / \mathrm{ml}$} & $<6$ & 34 & 4 & 30 & \multirow[t]{2}{*}{1.000} \\
\hline & $\geq 6$ & 22 & 3 & 19 & \\
\hline \multirow[t]{2}{*}{ CYFRA21-1, ng/ml } & $<3.3$ & 17 & 1 & 16 & \multirow[t]{2}{*}{0.421} \\
\hline & $\geq 3.3$ & 39 & 6 & 33 & \\
\hline \multirow[t]{2}{*}{ NSE, ng/ml } & $<16.3$ & 33 & 3 & 30 & \multirow[t]{2}{*}{0.429} \\
\hline & $\geq 16.3$ & 23 & 4 & 19 & \\
\hline Ki-67 index & $<50 \%$ & 40 & 5 & 35 & 1.000 \\
\hline
\end{tabular}


NSCLC, non-small cell lung cancer; small cell lung cancer, SCLC

\section{Figures}
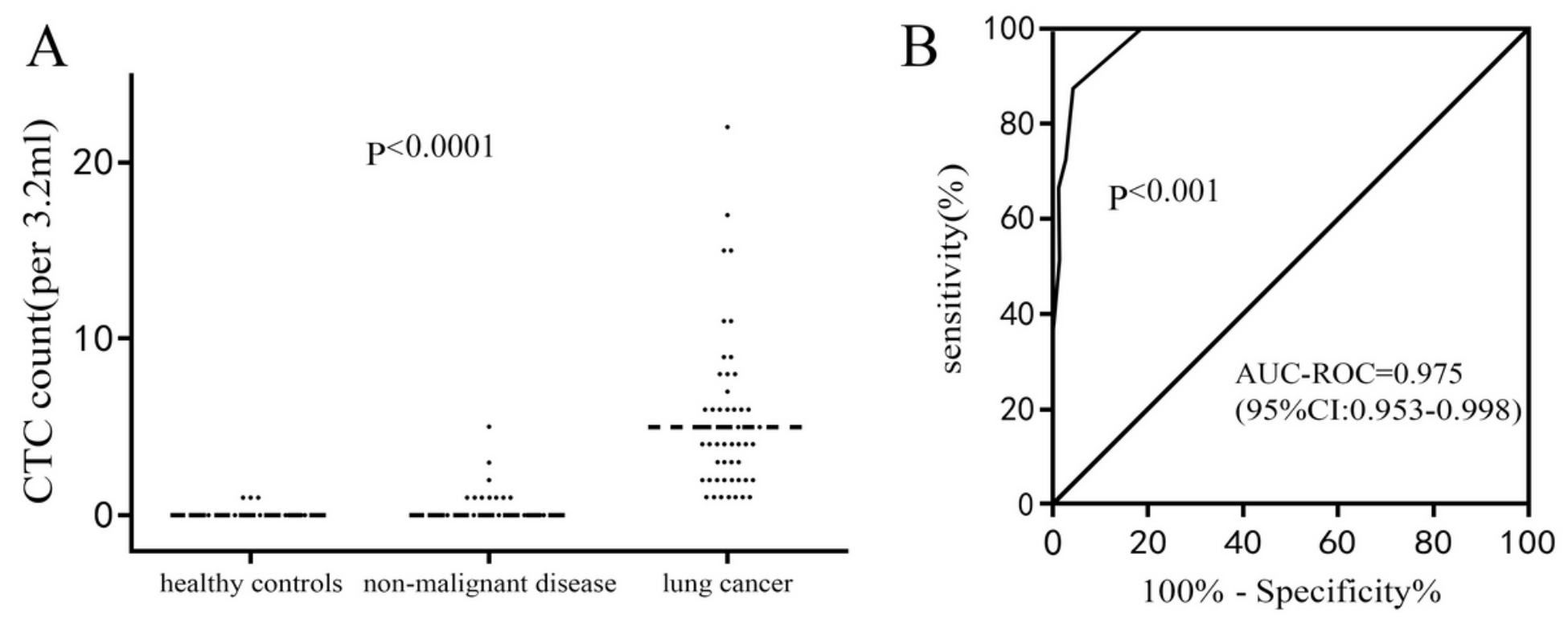

Figure 1

Detection of CTCs in LC patients, nonmalignant patients and healthy controls A. Distribution of CTCs count in healthy controls, nonmalignant patients and LC patients. B. The ROC curves for CTCs to discriminate LC from non-cancer controls.

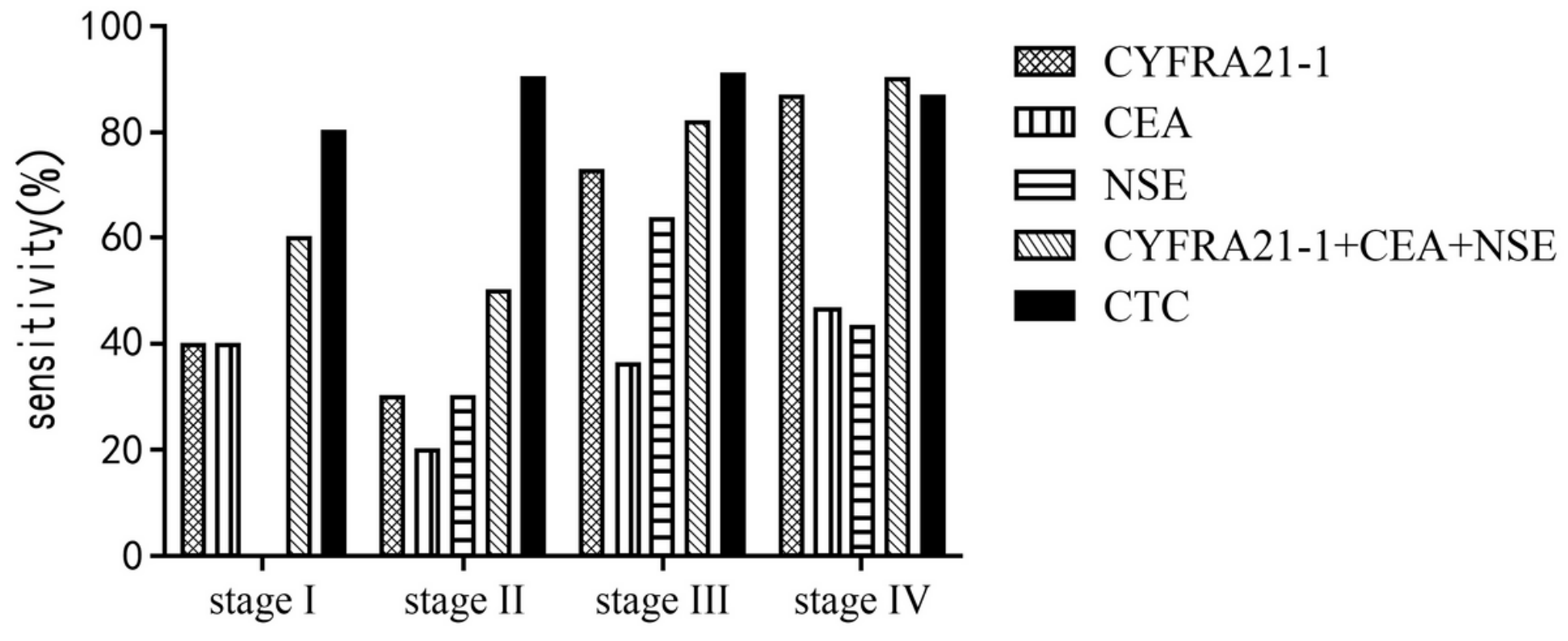

Figure 2

Detection sensitivity of CTCs and serum tumor markers in LC patients based on pathological stage 
A

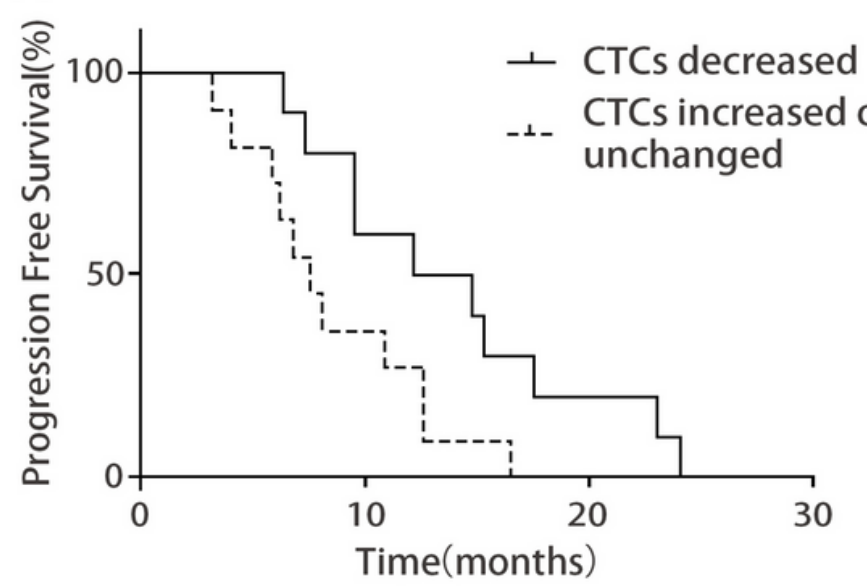

B

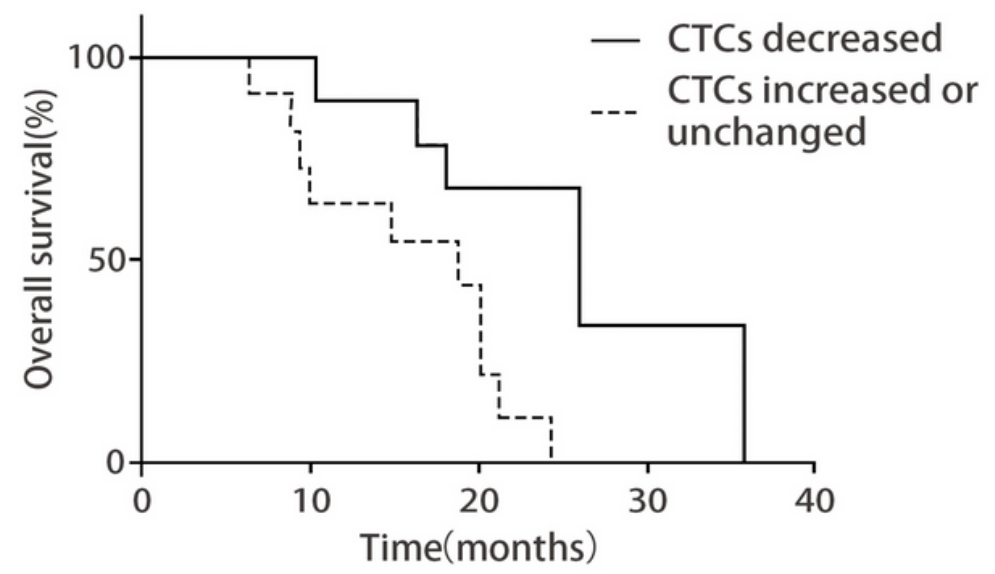

Figure 3

Kaplan-Meier curve for PFS and OS of 21 patients with SD 